PROCEEDINGS OF THE

AMERICAN MATHEMATICAL SOCIETY

Volume 136, Number 6, June 2008, Pages 2245-2252

S 0002-9939(08)09312-X

Article electronically published on February 14, 2008

\title{
RATIONAL HOMOTOPY TYPE OF SUBSPACE ARRANGEMENTS WITH A GEOMETRIC LATTICE
}

\author{
GERY DEBONGNIE \\ (Communicated by Paul Goerss)
}

\begin{abstract}
Let $\mathcal{A}=\left\{x_{1}, \ldots, x_{n}\right\}$ be a subspace arrangement with a geometric lattice such that $\operatorname{codim}(x) \geq 2$ for every $x \in \mathcal{A}$. Using rational homotopy theory, we prove that the complement $M(\mathcal{A})$ is rationally elliptic if and only if the sum $x_{1}^{\perp}+\ldots+x_{n}^{\perp}$ is a direct sum. The homotopy type of $M(\mathcal{A})$ is also given: it is a product of odd-dimensional spheres. Finally, some other equivalent conditions are given, such as Poincaré duality. Those results give a complete description of arrangements (with a geometric lattice and with the codimension condition on the subspaces) such that $M(\mathcal{A})$ is rationally elliptic, and show that most arrangements have a hyperbolic complement.
\end{abstract}

\section{INTRODUCTION}

Let $l$ be an integer. A subspace arrangement $\mathcal{A}$ is a finite set of affine subspaces in $\mathbb{C}^{l}$. We say that $\mathcal{A}=\left\{x_{1}, \ldots, x_{n}\right\}$ is central if all the affine subspaces $x_{i}$ are vector subspaces.

To every arrangement, we associate the set of nonempty intersections of elements of $\mathcal{A}$. This set $L(\mathcal{A})$ is partially ordered by $x \leq y \Longleftrightarrow y \subseteq x$. Let $x, y \in L(\mathcal{A})$. If $\mathcal{A}$ is central, we can define two operations on $L(\mathcal{A})$ : the meet $x \wedge y=\bigcap\{z \in L(\mathcal{A}) \mid$ $x \cup y \subset z\}$ and the join $x \vee y=x \cap y$. With these two operations, $L(\mathcal{A})$ is a lattice.

For every $x \in L(\mathcal{A})$, there exists a longest maximal chain $\mathbb{C}^{l}<x_{1}<\cdots<x_{r}=$ $x$. We say that the $\operatorname{rank}$ of $x, \operatorname{rk}(x)$, is $r$. The lattice $L(\mathcal{A})$ is called geometric if, for every $x, y \in L(\mathcal{A})$, we have $\operatorname{rk}(x)+\operatorname{rk}(y) \geq \operatorname{rk}(x \wedge y)+\operatorname{rk}(x \vee y)$.

The complement of a subspace arrangement $\mathcal{A}$ is the topological space

$$
M(\mathcal{A})=\mathbb{C}^{l} \backslash \bigcup \mathcal{A}
$$

In 2002, S. Yuzvinsky described a rational model for $M(\mathcal{A})$ in $[5$. Later on, in 6], S. Yuzvinsky and E. Feichtner proved that if the lattice $L(\mathcal{A})$ is geometric, then $M(\mathcal{A})$ is formal, and they give a simpler differential graded algebra model for $M(\mathcal{A})$.

Received by the editors January 31, 2007.

2000 Mathematics Subject Classification. Primary 55P62.

The author is an "Aspirant" of the "Fonds National pour la Recherche Scientifique" (FNRS), Belgium.

(C)2008 American Mathematical Society Reverts to public domain 28 years from publication 
In section 2. we state some basic properties of rational homotopy theory. The rational model defined by Yuzvinsky for $M(\mathcal{A})$ is recalled in section 3 . Arrangements with Poincaré duality are studied in section 4 . Finally, the main results are contained in section 5 ,

Briefly, Theorem 5.1 shows that, under some conditions, the following statements are equivalent: the subspace arrangement $\mathcal{A}$ has a rationally elliptic complement $M(\mathcal{A}), \operatorname{codim} \bigcap_{x \in \mathcal{A}} x=\sum \operatorname{codim} x, M(\mathcal{A})$ has Poincaré duality, and $M(\mathcal{A})$ has the homotopy type of a product of odd-dimensional spheres. Theorem 5.2 gives a geometric interpretation: these statements are equivalent to the fact that $x_{1}^{\perp}+\ldots+$ $x_{n}^{\perp}$ is a direct sum. So, every arrangement with a geometric lattice and rationally elliptic complement is obtained by taking a direct sum $y_{1} \oplus \ldots \oplus y_{n}$ of vector subspaces in $\mathbb{C}^{l}$ and then taking their orthogonal complement: $\mathcal{A}=\left\{y_{1}^{\perp}, \ldots, y_{n}^{\perp}\right\}$.

It shows that most arrangements have a hyperbolic complement. In that case, which is easy to check with the condition $\operatorname{codim} \bigcap_{x \in \mathcal{A}} x \neq \sum \operatorname{codim}(x)$, the sequence $\sum_{i \leq p}$ rk $\pi_{i}(M(\mathcal{A}))$ has an exponential growth and for any integer $N$, there are infinitely many $q$ with $\operatorname{rk} \pi_{q}(M(\mathcal{A})) \geq N$.

I would like to thank the referee for his/her work. In particular, the comments about the geometric interpretation were very helpful.

\section{RATIONAL HOMOTOPY THEORY}

For the basic facts on rational homotopy, we will refer to the classical references (see [4] or [3]).

Let $V$ be a graded vector space. The free commutative algebra on $V, \Lambda V$, is by definition the tensor product of the symmetric algebra on $V^{\text {even }}$ by the exterior algebra on $V^{\text {odd }}$. A minimal model is a differential graded algebra of the form $(\Lambda V, d)$ where $d(V) \subset \Lambda^{\geq 2} V$, and such that there is a basis of $V,\left(x_{a}\right)_{a \in A}$, indexed by a well-ordered set with the property that $d\left(x_{a}\right) \in \Lambda\left(x_{b}\right)_{b<a}$.

Each 1-connected space $X$ with finite Betti numbers admits a minimal model $(\Lambda V, d)$ that is unique up to isomorphism and that contains all the rational homotopy types of $X$. In particular, $\operatorname{dim} V^{n}=\operatorname{dim} \pi_{n}(X) \otimes \mathbb{Q}$.

Definition 2.1. The space $X$ is called formal if there is a quasi-isomorphism $(\Lambda V, d) \rightarrow\left(H^{\star}(X, \mathbb{Q}), 0\right)$.

In the case of subspace arrangements, it is known that if the lattice $L(\mathcal{A})$ is geometric, then the space $M(\mathcal{A})$ is formal (see [6]).

The dichotomy theorem in rational homotopy theory states that finite 1-connected $\mathrm{CW}$-complexes are either elliptic or hyperbolic, with the following properties: if $X$ is elliptic, then $\pi_{n}(X)=0$ for $n$ large enough and $H^{\star}(X ; \mathbb{Q})$ satisfies Poincaré duality. If $X$ is hyperbolic, then the sequence $\operatorname{dim} \pi_{n}(X)$ has an exponential growth.

We will use the following theorem (see [2] for details).

Theorem 2.2 (Félix-Halperin). If a space $X$ is elliptic and formal, then its minimal model has the form $(\Lambda V, d)=\left(\Lambda V_{0} \oplus V_{1}, d\right)$ with $V_{0}=V_{0}^{\text {odd }} \oplus V_{0}^{\text {even }}$, $\operatorname{dim} V_{0}^{\text {even }}=$ $\operatorname{dim} V_{1}, V_{1}=V_{1}^{\text {odd }}, d V_{0}=0$ and $d V_{1} \subset \Lambda V_{0}$. Moreover, the injection $\left(\Lambda V_{0}^{\text {odd }}, 0\right) \rightarrow$ $(\Lambda V, d)$ induces an injective map in cohomology.

Finally, to use rational homotopy theory, we need spaces that are 1-connected. The following lemmas will show that the space $M(\mathcal{A})$ is 1-connected if the subspaces all have a codimension $\geq 2$. 
Lemma 2.3. Let $\mathcal{A}=\left\{x_{1}, \ldots, x_{q}\right\}$ be a central arrangement in $\mathbb{C}^{l}$ such that $\operatorname{codim} x_{i} \geq 2$. Let $y_{i}=x_{i} \cap S^{2 l-1}$ and $f: S^{1} \rightarrow S^{2 l-1} \backslash \bigcup_{i=1}^{q} y_{i}$ be a smooth map. Then $f$ extends to a map $\bar{f}: D^{2} \rightarrow S^{2 l-1} \backslash \bigcup_{i=1}^{q} y_{i}$ :

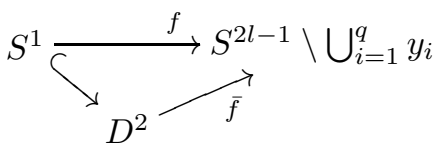

Proof. The proof is done by induction on $q$. The case $q=1$ is a direct consequence of Corollary 15.7 in [1]. Let's assume the result true until $q-1$. Let $f: S^{1} \rightarrow$ $S^{2 l-1} \backslash \bigcup_{i=1}^{q} y_{i}$. By induction, we know that there exists an $\tilde{f}: D^{2} \rightarrow S^{2 l-1} \backslash \bigcup_{i=1}^{q-1} y_{i}$ such that $\left.\tilde{f}\right|_{S^{1}}=f$. Let $r>0$ such that $r<\operatorname{dist}\left(\tilde{f}\left(D^{2}\right), \bigcup_{i=1}^{q-1} y_{i}\right)$ and

$$
T=\left\{z \in S^{2 l-1} \mid \operatorname{dist}\left(z, \bigcup_{i=1}^{q-1} y_{i}\right)<r\right\} .
$$

The way we constructed $T$ implies that $\operatorname{im} \tilde{f} \subset S^{2 l-1} \backslash T$, which is a $(2 l-1)$ dimensional manifold with boundary. In it, $y_{q} \backslash T$ is a compact submanifold of dimension $<2 l-4$ (because it is of codimension $\geq 2$ in $\mathbb{C}^{l}$ ). Corollary 15.6 in [1] gives the existence of a smooth map $\bar{f}: D^{2} \rightarrow S^{2 l-1} \backslash T$ such that $\left.\bar{f}\right|_{S^{1}}=\left.\tilde{f}\right|_{S^{1}}=f$ and $\bar{f}\left(D^{2}\right)$ is transverse to $y_{q} \backslash T$. But $\operatorname{dim} \bar{f}\left(D^{2}\right)+\operatorname{dim}\left(y_{q} \backslash T\right) \leq 2+2 l-4<2 l-1$. So, transversality can only happen if $\bar{f}\left(D^{2}\right) \cap\left(y_{q} \backslash T\right)=\emptyset$. Therefore, the application $\bar{f}\left(D^{2}\right)$ is such that $\operatorname{im} \bar{f}\left(D^{2}\right) \subset\left(S^{2 l-1} \backslash T\right) \backslash\left(y_{q} \backslash T\right) \subset S^{2 l-1} \backslash \bigcup_{i=1}^{q} y_{i}$.

Lemma 2.4. Let $\mathcal{A}$ be a subspace arrangement such that for each $x \in \mathcal{A}, \operatorname{codim} x \geq$ 2. Then the space $M(\mathcal{A})$ is 1-connected.

Proof. Let $f: S^{1} \rightarrow M(\mathcal{A})$ be a map. Since the $x_{i}$ are vector spaces, we can define the homotopy $h_{t}=(1-t) f+t \frac{f}{\|f\|}$. We can assume that the map $h_{1}: S^{1} \rightarrow$ $S^{2 l-1} \backslash\left(\bigcup_{x \in \mathcal{A}}\left(x \cap S^{2 l-1}\right)\right)$ is smooth. So, Lemma 2.3 can be applied and shows that $f \simeq h_{1} \simeq \star$. This implies that $\pi_{1}(M(\mathcal{A}))=0$, so $M(\mathcal{A})$ is 1-connected.

\section{RAtional MODEL of SUbSPACE ARRANGEMENTS}

Let $\mathcal{A}$ be a central arrangement of subspaces in $\mathbb{C}^{l}$. Yuzvinsky defined the relative atomic differential graded algebra $D_{\mathcal{A}}=(D, d)$ associated with an arrangement as follows (see [6]): choose a linear order on $\mathcal{A}$. The chain complex $(D, d)$ is generated by all subsets $\sigma \subseteq \mathcal{A}$. For $\sigma=\left\{x_{1}, \ldots, x_{n}\right\}$, we define the differential by

$$
d \sigma=\sum_{j: \vee\left(\sigma \backslash\left\{x_{j}\right\}\right)=\vee \sigma}(-1)^{j}\left(\sigma \backslash\left\{x_{j}\right\}\right)
$$

where the indexing of the elements in $\sigma$ follows the linear order imposed on $\mathcal{A}$. With $\operatorname{deg}(\sigma)=2 \operatorname{codim} \vee \sigma-|\sigma|,(D, d)$ is a cochain complex. Finally, we need a multiplication on $(D, d)$. For $\sigma, \tau \subseteq \mathcal{A}$,

$$
\sigma \cdot \tau= \begin{cases}(-1)^{\operatorname{sgn} \epsilon(\sigma, \tau)} \sigma \cup \tau, & \text { if } \operatorname{codim} \vee \sigma+\operatorname{codim} \vee \tau=\operatorname{codim} \vee(\sigma \cup \tau), \\ 0, & \text { otherwise }\end{cases}
$$

where $\epsilon(\sigma, \tau)$ is the permutation that, applied to $\sigma \cup \tau$ with the induced linear order, places elements of $\tau$ after elements of $\sigma$, both in the induced linear order. 
A subset $\sigma \subseteq \mathcal{A}$ is said to be independent if $\operatorname{rk}(\vee \sigma)=|\sigma|$. When $\mathcal{A}$ is an arrangement with a geometric lattice, we have the following property: $H^{\star}(M(\mathcal{A}))$ is generated by the classes $[\sigma]$, with $\sigma$ independent ([6]).

\section{Poincaré duality}

Having Poincaré duality is a strong statement for a subspace arrangement with geometric lattice. That condition alone determines the minimal model of the complement.

For $\mathcal{A}=\left\{x_{1}, \ldots, x_{n}\right\}$ a subspace arrangement with $L(\mathcal{A})$ geometric, let $M_{r}$ be the greatest element in $L(\mathcal{A})$ and $\mathbb{C}^{l}<M_{1}<\ldots<M_{r-1}<M_{r}$ be any maximal chain in $L(\mathcal{A})$. In particular, $\operatorname{rk}\left(M_{i}\right)=i$ for $1 \leq i \leq r$. Let $X_{i}=\left\{x \in \mathcal{A} \mid x<M_{i}\right\}$. We can construct a chain complex $C_{\star}^{i}: C_{p}^{i}$ is the module generated by all the linear combinations of the $\sigma \subset \mathcal{A}$ such that $\vee \sigma=M_{i}$ and $|\sigma|=p$. With the differential defined in the Yuzvinsky model of $M(\mathcal{A})$ and $\operatorname{deg}(\sigma)=|\sigma|, C_{\star}^{i}$ is clearly a chain complex.

Lemma 4.1. Let $\mathcal{A}=\left\{x_{1}, \ldots, x_{n}\right\}$ be a subspace arrangement with geometric lattice. If $M(\mathcal{A})$ has Poincaré duality and $1 \leq k \leq r$, then $\operatorname{dim} H_{k}\left(C_{\star}^{k}\right)=1$.

Proof. In this proof, $X_{0}$ is the empty set. Let $E_{p}^{i}(1 \leq i \leq r)$ be the submodule of $C_{p}^{i}$ generated by

- all the $\sigma \subset \mathcal{A}$ such that $|\sigma|=p, \vee \sigma=M_{i}$ and $\sigma$ contains at least 2 elements of $X_{i} \backslash X_{i-1}$,

- all the elements $\left\{x_{i_{1}}, x_{i_{2}}, \ldots, x_{i_{p-1}}, y_{1}\right\}-\left\{x_{i_{1}}, \ldots, x_{i_{p-1}}, y_{2}\right\}$ with $x_{i_{j}} \in$ $X_{i-1}$ and $y_{1}, y_{2} \in X_{i} \backslash X_{i-1}$.

It is easy to check that $E_{\star}^{i}$ is a subcomplex of $C_{\star}^{i}$. We have the following short exact sequences:

$$
0 \rightarrow E_{\star}^{i} \rightarrow C_{\star}^{i} \rightarrow C_{\star}^{i} / E_{\star}^{i} \rightarrow 0 .
$$

Since $\mathcal{A}$ has a geometric lattice, the cohomology is generated by the classes $\sigma \in \mathcal{A}$ such that $\sigma$ is independent. So, the (reduced) homology of $C_{\star}^{i}$ is 0 in every degree except possibly the $i^{\text {th }}$ degree. We have $H_{\star}\left(C_{\star}^{i}\right)=H_{i}\left(C_{\star}^{i}\right)$. For $1 \leq i \leq r$, we know that $\operatorname{rk}\left(M_{i}\right)=i$. This means that $E_{i}^{i+1}$ is an empty set and $H_{i}\left(E_{\star}^{i+1}\right)=0$. Hence, the long exact sequence in homology associated with the short exact sequence above implies that the map $H_{i+1}\left(C_{\star}^{i+1}\right) \rightarrow H_{i+1}\left(C_{\star}^{i+1} / E_{\star}^{i+1}\right)$ is surjective.

Since $X_{i+1} \backslash X_{i}$ is nonempty (because $\vee X_{i+1}=M_{i+1}$ and $\vee X_{i}=M_{i}$ ), we can fix some $y \in X_{i+1} \backslash X_{i}$. This $y$ define maps $\varphi_{p}: C_{p}^{i} \rightarrow C_{p+1}^{i+1} / E_{p+1}^{i+1}$ sending $\left\{x_{1}, \ldots, x_{p}\right\}$ to $\left[\left\{x_{1}, \ldots, x_{p}, y\right\}\right]$. Since the lattice is geometric, if $\vee\left\{x_{1}, \ldots, \hat{x}_{j}, \ldots, x_{p}\right\}<M_{i}$, then $\vee\left\{x_{1}, \ldots, \hat{x_{j}}, \ldots, x_{p}, y\right\}<M_{i+1}$. Therefore, the maps $\varphi_{p}$ commute with the differentials and define an isomorphism of chain complexes $\left(C_{\star}^{i}\right)_{p} \rightarrow\left(C_{\star}^{i+1} / E_{\star}^{i+1}\right)_{p+1}$. Hence, $H_{p}\left(C_{\star}^{i}\right)=H_{p+1}\left(C_{\star}^{i+1} / E_{\star}^{i+1}\right)$. But, we proved that the map $H_{i+1}\left(C_{\star}^{i+1}\right) \rightarrow$ $H_{i+1}\left(C_{\star}^{i+1} / E_{\star}^{i+1}\right)$ is a surjection. So

$$
\operatorname{dim} H_{i}\left(C_{\star}^{i}\right)=\operatorname{dim} H_{i+1}\left(C_{\star}^{i+1} / E_{\star}^{i+1}\right) \leq \operatorname{dim} H_{i+1}\left(C_{\star}^{i+1}\right) .
$$

Since the space $M(\mathcal{A})$ has Poincare duality, there is a unique cohomology class in the highest degree in $H^{\star}\left(D_{\mathcal{A}}\right)$. That cohomology class is represented by an independent $\sigma \in \mathcal{A}$ such that $|\sigma|=r$. If $\operatorname{dim} H_{r}\left(C_{\star}^{r}\right) \geq 2$, then there is another class $[\tau]$ and by Poincaré duality, there is an element $[\rho]$ in $H^{\star}\left(D_{\mathcal{A}}\right)$ such that $[\sigma]=[\rho][\tau]$, but this 
is impossible by the multiplication law because $\operatorname{codim} \vee \sigma=\operatorname{codim} \vee \tau$. Therefore, $\operatorname{dim} H_{r}\left(C_{\star}^{r}\right)=1$. From the following sequence of inequalities,

$$
1=\operatorname{dim} H_{1}\left(C_{\star}^{1}\right) \leq \operatorname{dim} H_{2}\left(C_{\star}^{2}\right) \leq \cdots \leq \operatorname{dim} H_{r}\left(C_{\star}^{r}\right)=1,
$$

we deduce that $\operatorname{dim} H_{k}\left(C_{\star}^{k}\right)=1$ for all $1 \leq k \leq r$.

Lemma 4.2. Let $\mathcal{A}=\left\{x_{1}, \ldots, x_{n}\right\}$ be a subspace arrangement such that $L(\mathcal{A})$ is geometric and $M(\mathcal{A})$ has Poincaré duality. Let $M \in L(\mathcal{A})$ with $\operatorname{rk}(M)=i$ and let $X(M)=\{x \in \mathcal{A} \mid x \leq M\}$. Then \#X(M)=i.

Proof. We prove the result by induction on $\operatorname{rk} M$. It is clear for $i=1$. Now, let us suppose that it is true for all $N \in L(\mathcal{A})$ with $\operatorname{rk} N \leq i-1$ and let $M \in L(\mathcal{A})$ with $\operatorname{rk} M=i$. Denote by $M_{1}<M_{2}<\ldots<M_{i}=M<M_{i+1}<\ldots<M_{r}$ a maximal sequence in $L(\mathcal{A})$, and write

$$
X\left(M_{i-1}\right)=\left\{x_{1}, \ldots, x_{i-1}\right\} \quad \text { and } \quad X(M)=\left\{x_{1}, \ldots, x_{i-1}, x_{i-1+1}, \ldots, x_{i-1+l}\right\} .
$$

We consider the chain complex $C_{\star}^{i}$ defined at the beginning of this section for that maximal chain. Remark first that if $\left\{x_{n_{1}}, \ldots, x_{n_{i+1}}\right\} \subset X(M)$ with $\vee x_{n_{i}}=M$, then for each $k, \vee\left\{x_{n_{1}}, \ldots, \hat{x}_{n_{k}}, \ldots, x_{n_{i+1}}\right\}=M$, because otherwise $\vee\left\{x_{n_{1}}, \ldots, \hat{x}_{n_{k}}, \ldots\right.$, $\left.x_{n_{i+1}}\right\}$ is an element $N$ in $L(\mathcal{A})$ with $\operatorname{rk} N<i$. So, there are $i$ subspaces $y_{j}$ with $y_{j}<$ $N$, in contradiction with our induction hypothesis. Therefore, if $\left\{x_{n_{1}}, \ldots, x_{n_{i+1}}\right\} \subset$ $C_{i+1}^{i}$, then

$$
d\left\{x_{n_{1}}, \ldots, x_{n_{i+1}}\right\}=\sum_{j=1}^{i+1}(-1)^{j}\left\{x_{n_{1}}, \ldots, \hat{x}_{n_{j}}, \ldots, x_{n_{i+1}}\right\} .
$$

In the complex $C_{\star}^{i}$, every cycle of degree $i$ is equivalent to a sum $\sum \alpha_{j}\left\{x_{1}, x_{j_{2}}, \ldots\right.$, $\left.x_{j_{i}}\right\}$. Indeed, if $1 \notin\left\{j_{1}, \ldots, j_{i}\right\}$, then

$$
\left\{x_{j_{1}}, \ldots, x_{j_{i}}\right\}=-d\left\{x_{1}, x_{j_{1}}, \ldots, x_{j_{i}}\right\}+\sum_{k=2}^{i}(-1)^{k}\left\{x_{1}, \ldots, \hat{x}_{j_{k}}, \ldots, x_{j_{i}}\right\} .
$$

Now, no cycle of the form $\sum_{j} \alpha_{j}\left\{x_{1}, x_{j_{2}}, \ldots, x_{j_{i}}\right\}$ is a boundary. Suppose this is the case. Then we have

$$
\sum_{j} \alpha_{j}\left\{x_{1}, x_{j_{2}}, \ldots, x_{j_{i}}\right\}=d\left[\sum_{m} \beta_{m}\left\{x_{1}, x_{m_{1}}, \ldots, x_{m_{i}}\right\}+\sum_{n} \gamma_{n}\left\{x_{n_{1}}, \ldots, x_{n_{i+1}}\right\}\right]
$$

with $1 \notin\left\{x_{1}, \ldots, n_{i+1}\right\}$. Developing the differential, we get

$$
0=-\sum_{m} \beta_{m}\left\{x_{m_{1}}, \ldots, x_{m_{i}}\right\}+\sum_{n} \gamma_{n}\left(\sum_{k=1}^{i+1}(-1)^{k}\left\{x_{n_{1}}, \ldots, \hat{x}_{n_{k}}, \ldots, x_{n_{i+1}}\right\}\right) \text {. }
$$

We deduce that

$$
\begin{aligned}
& d\left(\sum_{n} \gamma_{n}\left\{x_{1}, x_{n_{1}}, \ldots, x_{n_{i+1}}\right\}\right) \\
& =-\sum_{n} \gamma_{n}\left\{x_{n_{1}}, \ldots, x_{n_{i+1}}\right\}-\sum_{n} \gamma_{n}\left(\sum_{k=1}^{i+1}(-1)^{k}\left\{x_{1}, x_{n_{1}}, \ldots, \hat{x}_{n_{k}}, \ldots, x_{n_{i+1}}\right\}\right) \\
& =-\sum_{n} \gamma_{n}\left\{x_{n_{1}}, \ldots, x_{n_{i+1}}\right\}-\sum_{m} \beta_{m}\left\{x_{1}, x_{m_{1}}, \ldots, x_{m_{i}}\right\} .
\end{aligned}
$$


Since $d^{2}=0$, this gives $\sum_{j} \alpha_{j}\left\{x_{1}, x_{j_{2}}, \ldots, x_{j_{i}}\right\}=0$.

We deduce from the above calculation that $l=1$, i.e. $X(M)=\left\{x_{1}, \ldots, x_{i}\right\}$, because otherwise, the cycles $\left\{x_{1}, \ldots, x_{i}\right\}$ and $\left\{x_{1}, \ldots, x_{i-1}, x_{i+1}\right\}$ would be linearly independant in homology, in contradiction with Lemma 4.1

Proposition 4.3. Let $\mathcal{A}=\left\{x_{1}, \ldots, x_{n}\right\}$ be a subspace arrangement. If $L(\mathcal{A})$ is geometric and $M(\mathcal{A})$ has Poincaré duality, then the minimal model of $M(\mathcal{A})$ is the algebra $\left(\Lambda\left(y_{1}, \ldots, y_{n}\right), 0\right)$ where $\operatorname{deg} y_{i}=2 \operatorname{codim} x_{i}-1$.

Proof. This is a consequence from Lemma 4.2 This lemma shows that every subset $\sigma \subset \mathcal{A}$ is independent. Therefore, any product $\left\{x_{i_{1}}\right\} \cdot \ldots \cdot\left\{x_{i_{l}}\right\} \neq 0$, and all the products are different in cohomology.

\section{MAIN RESULtS}

Now, everything is in place to prove the main results. The first theorem uses rational homotopy theory and gives some equivalent conditions to the fact that $M(\mathcal{A})$ is rationally elliptic. With some linear algebra, the second theorem shows that the condition (3) has a geometric interpretation in term of the orthogonal subspaces $x_{i}^{\perp}$.

Theorem 5.1. Let $\mathcal{A}$ be a subspace arrangement with a geometric lattice such that every $x \in \mathcal{A}$ has $\operatorname{codim}(x) \geq 2$. Then the following conditions are equivalent:

(1) $M(\mathcal{A})$ is rationally elliptic,

(2) $M(\mathcal{A})$ has the rational homotopy type of a product of odd-dimensional spheres,

(3) $\operatorname{codim} \bigcap_{x \in \mathcal{A}} x=\sum \operatorname{codim} x$,

(4) $M(\mathcal{A})$ has the homotopy type of a product of odd-dimensional spheres,

(5) $M(\mathcal{A})$ has Poincaré duality.

Proof. (1) implies (2). Since $L(\mathcal{A})$ is geometric, we know (see [6]) that $M(\mathcal{A})$ is a formal space. If $M(\mathcal{A})$ is elliptic, we can apply Theorem [2.2, By definition of the differential, every $x \in \mathcal{A},\{x\}$ is a generator in cohomology for the rational model described in section 3 . The degree of $[\{x\}]$ is $2 \operatorname{codim} x-1$. Therefore, the $(\{x\})_{x \in \mathcal{A}}$ form a linearly independent sequence in $V_{0}^{\text {odd }}$. By Theorem 2.2, we have an injective map

$$
\rho: \Lambda_{x \in \mathcal{A}}[\{x\}] \rightarrow H^{\star}(\Lambda V) .
$$

In particular, for each sequence $x_{1}, \ldots, x_{n}$ in $\mathcal{A}$, with $x_{i} \neq x_{j}$, we have

$$
\left\{x_{1}\right\} \cdot\left\{x_{2}\right\} \cdot \ldots \cdot\left\{x_{n}\right\} \neq 0
$$

because their product is nonzero in cohomology. Therefore, we have the following equality: $\prod_{i=1}^{n}\left\{x_{i}\right\}= \pm\left\{x_{1}, x_{2}, \ldots, x_{n}\right\}$ and $\left[\left\{x_{1}, \ldots, x_{n}\right\}\right] \neq 0$ (in cohomology).

The map $\rho$ is surjective because, for each independent set $\left\{x_{1}, \ldots, x_{n}\right\}$ (which generates $\left.H^{\star}(M(\mathcal{A}))\right)$, we have $\left[\left\{x_{1}, \ldots, x_{n}\right\}\right]= \pm \prod_{i=1}^{n}\left[\left\{x_{i}\right\}\right]$, which is in the image of $\rho$. This implies that the map $\rho$ is an isomorphism. By Lemma 2.4 $M(\mathcal{A})$ is 1-connected. Therefore, $M(\mathcal{A})$ has the rational homotopy type of a product of odd-dimensional spheres.

(2) implies (3). We showed that the product $\prod_{x \in \mathcal{A}}\{x\} \neq 0$. By definition of the product, this implies that $\operatorname{codim} \bigcap_{x \in \mathcal{A}} x=\sum_{x \in \mathcal{A}} \operatorname{codim} x$.

(3) implies (4). Let $\mathcal{A}=\left\{x_{1}, \ldots, x_{n}\right\}$ be a subspace arrangement in $\mathbb{C}^{l}$ such that $\operatorname{codim} \bigcap x_{i}=\sum \operatorname{codim} x_{i}$. The quotient map $p: \mathbb{C}^{n} \rightarrow \mathbb{C}^{n} /\left(\bigcap x_{i}\right)$ induces a 
homotopy equivalence $\left(\mathbb{C}^{n} \backslash \bigcup x_{i}\right) \rightarrow\left(\left(\mathbb{C}^{n} / \bigcap x_{i}\right) \backslash \bigcup\left(x_{i} / \bigcap x_{i}\right)\right)$. Hence we can assume that $\bigcap x_{i}=0$.

Let's write $x_{i}=\operatorname{ker}\left(H_{i}: \mathbb{C}^{n} \rightarrow \mathbb{C}^{n_{i}}\right)$. The map

$$
\left(H_{1}, H_{2}, \ldots, H_{n}\right): \mathbb{C}^{n} \rightarrow \prod \mathbb{C}^{n_{i}}
$$

is an isomorphism, which induces a homotopy equivalence

$$
\mathbb{C}^{n} \backslash \bigcup x_{i} \rightarrow \prod_{i=1}^{n}\left(\mathbb{C}^{n_{i}} \backslash\{0\}\right) .
$$

But the injective map $\prod\left(S^{2 n_{i}-1}\right) \rightarrow \prod\left(\mathbb{C}^{n_{i}} \backslash\{0\}\right)$ is a homotopy equivalence. Therefore $M(\mathcal{A})=\mathbb{C}^{n} \backslash \bigcup x_{i}$ has the homotopy type of a product of odd-dimensional spheres.

(4) implies (1). Obvious.

(1) implies (5). Obvious.

(5) implies (2). Direct consequence from Proposition 4.3.

Theorem 5.2. Let $\mathcal{A}=\left\{x_{1}, \ldots, x_{n}\right\}$ be a subspace arrangement. Then the following conditions are equivalent:

(1) $\operatorname{codim} \bigcap_{i=1}^{n} x_{i}=\sum_{i=1}^{n} \operatorname{codim} x_{i}$,

(2) the sum $x_{1}^{\perp}+\ldots+x_{n}^{\perp}$ is a direct sum.

Proof. First, let's prove by induction on $k, 2 \leq k \leq n$, that :

$$
\left(\bigcap_{j=1}^{k} x_{j}\right)^{\perp}=\sum_{i=1}^{k} x_{i}^{\perp}
$$

For $k=2$, it gives $\left(x_{1} \cap x_{2}\right)^{\perp}=x_{1}^{\perp}+x_{2}^{\perp}$, which is a well-known fact. Now, let's suppose that the formula is true until $k-1$. We have:

$$
\left(\bigcap_{j=1}^{k} x_{j}\right)^{\perp}=\left(\bigcap_{j=1}^{k-1} x_{j} \cap x_{k}\right)^{\perp}=\left(\bigcap_{j=1}^{k-1} x_{j}\right)^{\perp}+x_{k}^{\perp} .
$$

Using the induction hypothesis concludes the proof. Now, we can prove the theorem:

$$
\begin{aligned}
& x_{1}^{\perp}+\ldots+x_{n}^{\perp} \text { is a direct sum } \Longleftrightarrow \sum_{i=1}^{n} \operatorname{dim} x_{i}^{\perp}=\operatorname{dim}\left(\sum_{i=1}^{n} x_{i}^{\perp}\right) \\
& \Longleftrightarrow \sum_{i=1}^{n} \operatorname{codim} x_{i}=\operatorname{dim}\left(\bigcap_{i=1}^{n} x_{i}\right)^{\perp} \Longleftrightarrow \sum_{i=1}^{n} \operatorname{codim} x_{i}=\operatorname{codim} \bigcap_{i=1}^{n} x_{i} .
\end{aligned}
$$

\section{REFERENCES}

1. G. Bredon, Topology and Geometry, Graduate Texts in Mathematics, 139, Springer-Verlag, 1993. MR 1224675 (94d:55001)

2. Y. Félix, S. Halperin, Formal spaces with finite-dimensional rational homotopy, Trans. Amer. Math. Soc. 270 (1982), no. 2, 575-588. MR645331 (83h:55023)

3. Y. Félix, S. Halperin, J.-C. Thomas, Rational Homotopy Theory, Graduate Texts in Mathematics, 205, Springer-Verlag, 2000. MR1802847 (2002d:55014)

4. D. Sullivan, Infinitesimal computations in topology, Publ. IHES 47 (1977), 269-331. MR0646078 (58:31119) 
5. S. Yuzvinsky, Small rational model of subspace complement, Trans. Amer. Math. Soc. 354 (2002), no. 5, 1921-1945. MR1881024 (2003a:52030)

6. E. Feichtner, S. Yuzvinsky, Formality of the complements of subspace arrangements with geometric lattices, J. Math. Sci. (N.Y.) 140 (2007), 472-479. MR2183223 (2007a:55021)

Université Catholique de louvain, Departement de Mathematique, Chemin du Cyclotron, 2, B-1348 Louvain-la-Neuve, Belgium

E-mail address: debongnie@math.ucl.ac.be 